\title{
Design and Implementation of Motion Controller Based on Dual core architecture
}

\author{
Liu yun ${ }^{1, a}$, Liu peng ${ }^{2, b}$ and Liu an cai ${ }^{3, c}$ \\ ${ }^{1}$ Chong Qing Energy College, Chong Qing, China \\ ${ }^{2}$ Chong Qing Communication College, China \\ ${ }^{3}$ Chong Qing Energy College, Chong Qing, China

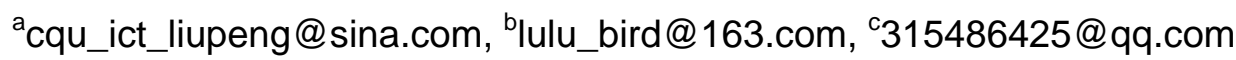

Keywords: Motion-control; High precision CNC machine tools; ARM+FPGA; adaptive pid algorithm

\begin{abstract}
This paper innovatively presents ARM9 processor-AT91RM9200 and CYCLONE series FPGA-EP3C16F484 as core to design motion control system for high precision CNC machine tools. This paper introduces key techniques of motion control system in details from hardware to software, using adaptive pid algorithm based on single neuron as motion control algorithm in the system, then give simulation result of adaptive pid algorithm based on single neuron, at last list the system operation results.
\end{abstract}

\section{Introduction}

Motion control system of High precision CNC machine tools usually need to provide accuracy and real-time control to multi-axis motion system ${ }^{[1,2]}$. The former system mostly uses Industrial Personal Computer(IPC) and multi control cards to build up Motion Controller. High precision CNC machine tools have more strictly requirements in size, cost, power consumption, real-time response and reliability, accompanying with its extent and complexity increased. on the other hand the number of axies increased, demand of control accuracy is increasingly high, real-time demand of controlled object grows stronger, currently existing motion control system based on IPC+ multi control cards can't satisfy the request with the future system ${ }^{[3,4]}$.

The controller based on ARM+FPGA offers higher performance than that with IPC+ multi control cards. This paper builds up the hardware architecture of the motion control system with ARM+FPGA. ARM9 processor is rich in hardware and software resources, assuring full-function of motion control system, moreover, parallel processing capability and reconfigurable feature of FPGA assures not only the decoupling ability, but also the reconfigurability of the multi-axis controller.

\section{Key Technology of Hardware System}

Motion control system based on ARM+FPGA have several advantages over currently existing motion control system based on IPC+multi control cards ${ }^{[5]}$ :

Design philosophy of Soft Hardware. The controller based on ARM+FPGA use FPGA to implement details of motion control task, not only belongs to reconfigurability of software, but also high integrative performance and reliability of hardware. Embedded applications programme has many obvious advantages mainly reflected in size and cost, compared to currently existing motion control system based on IPC+ multi control cards.

Global design of hardware system.The Overall Block Diagram of Motion Controller for High precision CNC machine tools is shown in Figure1. The motion controller take ARM9 processor-AT91RM9200 and Cyclone FPGA-EP3C16F484as core. The AT91RM9200 is the master chip of the system, it takes charge of the system Control, at the same time, it exchanges Data between FPGA and itself with AHB. When the AT91RM9200 receives enough data from FPGA, it transmit the data blocks to host Industrial Personal Computer with Network Protocol, at the last, the host Industrial Personal Computer complete the processing and reconstruction of the image. In the 
system the FPGA take charge of receiving the collected serial data from Data Acquisition System, it transforms the serial data into parallel data, and store the parallel data into ram configured in FPGA, afterwards, it send interrupt to arm in order to inform AT91RM9200 to read data from ram configured in FPGA. The arm9 system and the FPGA system connect each other with High-speed AHB bus in order to transmit data in high speed ${ }^{[6]}$.

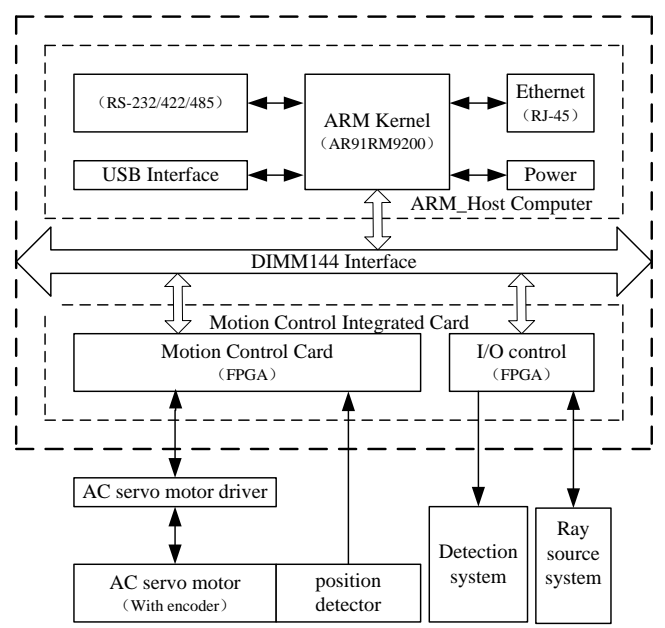

Figure 1. Overall Block Diagram of Motion Controller for High precision CNC machine tools.

Interface between ARM and FPGA.In the motion control system, the arm9 processor take the role of General Processor, its major function include system task triggering, system command transmitting, task scheduling and so on. The Cyclone FPGA in the system acts as a peripheral of ARM9 processor, it transmits or count pulse according to arm command transmitted by Data and Address Buses from arm.FPGA1 and FPGA2 connect to arm with each other's Data and Address Bus, Two-way data exchange is permitted between both sides.FPGA1 and FPGA2 acts as a peripheral of ARM9 processor, both of them Run in separate address space, ARM9 processor uses Address Strobe and address bus to Address the internal registers.

\section{Key Technologies of Software}

Design of the software based on the hierarchical design approach. The entire system software consists of Host operating system, Host development software, Target board operating system, Target board driver, Target board application, Composed of hardware description language HDL and so on. ARM9 processor in target system control Configuration registers of FPGA by driver, in order to execute control tasks. In the software of this paper, This paper centre on design of internal function modules and driver interface.

Internal key function module of FPGA.Single-axis control module shows in Figure 2. Two counters configured in each Location management unit: Logical location counter and actual location counter. The Logical location counter and actual location counter constitute a closed-loop system, the logical location counter is the input of the Closed-loop system, correspondingly, the actual location counter is the output of the Closed-loop system. When the range of error between the two counter is no more than a certain limit, it is not necessary to turn on motion control process, conversely, When the range of error between the two counter is more than a certain limit, The FPGA emit a interrupt request to $\mathrm{ARM}^{[7]}$. When ARM9 processor receive the interrupt, it turn on a motion control process, the motion control process tunes PID parameters of the motion control system, calculating controlling quantity ${ }^{[8]}$.

Interface of driver. The overall module of FPGA shows in Figure 3. In the system, FPGA1 and FPGA2 acts as a peripheral of ARM9 processor, both of them are bounded to peripheral space of ARM9 processor. In the system FPGA1 is mount on NCS2 space of ARM9 processor, its address space range from 0x30000000 to 0x3fffffff, FPGA2 is mount on NCS3 of ARM9 processor, its address space range from 0x40000000 to 0x4fffffff. In the load module of driver, all registers configured in FPGA are mapping to address space of ARM9 processor, as a result, arm take two 
FPGA as its peripheral chip, data transmission between arm9 processor and FPGA conducts by chip selection signal, address bus and data bus.

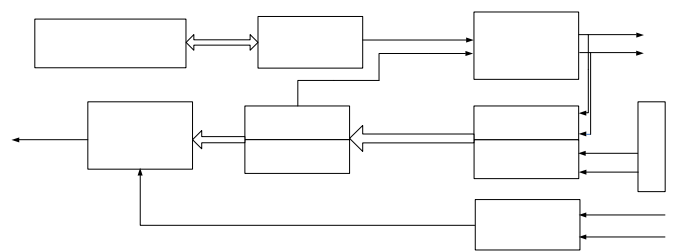

Figure 2. Single-axis control module.

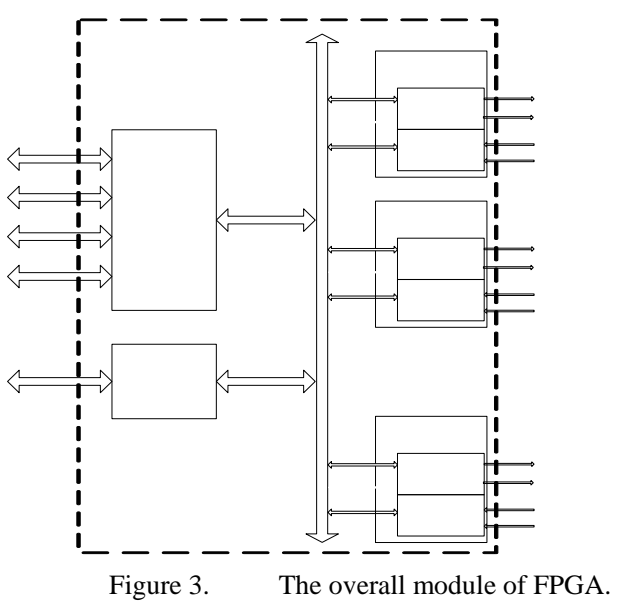

\section{Analysis of the Operation Results}

Position test results. To test position control effect of motion control system, do experiment with electrical motor for 200 times, each experimental data is the average value of the 200 experimental data. Fitting curve diagram of displacement test is shown in Figure 4. In the figure the blue line marked by '*' represents the pulse from generator, its number is set by FPGA, the red line marked by 'o' represents the pulse number read from counter, which is configured by FPGA. From the diagram it is concluded that:

a. Curve error of pulse generator and pulse counter accumulates gradually. When the error exceeds $0.18 \%$ of set value, The Adaptive pid algorithm of single-neuron starts to tune PID parameters, the tuning result make the error tend to 0 .

b. Error of pulse from counter is larger than that in generator, the reason of this result is that except the error from the generator, the error of the counter also impact on the system.

Speed test results.To test speed control effect of motion control system, do experiment with electrical motor for 200 times, each experimental data is the average value of 200 experimental data. Fitting curve diagram of speed test is shown in Figure 5. Set-speed is corresponding to Frequency coefficient of pulse generator in Figure 5.

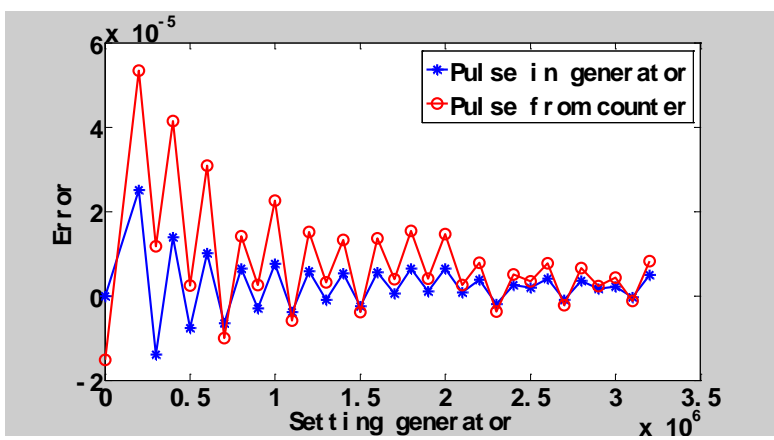

Figure 4.
Fitting curve diagram of displacement test.

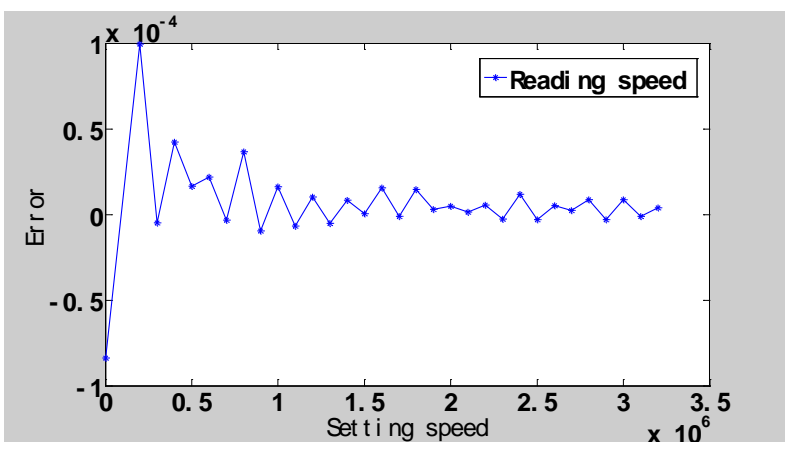

Figure 5. Fitting curve diagram of speed test.

\section{Conclusion}

The multi-axis common motion control system base on ARM and FPGA is a high quality advanced numerical control system which is integrated with computer numerical control technology, ARM technology, FPGA technology and embedded OS and so on, at the same time it is the first try in the domestic to research and develop low cost and high quality multi-axis numerical control system based on embedded system, it is significant in exploration in this subject. Tests and operation results indicate that the system has high measurement accuracy and good performance, 
and its design totally meets the project requirements. This paper accomplishes a basic exploration work for the further development of own intellectual property intelligent multi-axis motion controller based on embedded system.

\section{References}

[1] CHEN X S. The large-scale industrial CT'S data transfer system[J]. Nuclear Electronics\&Detection Technology, 2004, 24(4):56—58.

[2] ZHANG P, ZHOU R F. High speed multi-channel data acquisition system and storage system based on PCI bus[J]. Journal of Chongqing University: Natural Science E—dition, 2006, 29(3): 91-95.

[3] Guo Z P,Dong Y F,Zhang z z. Computerized tomography. Nondestructive Testing. $1996,18(1): 27-30)$

[4] Wang J P,Huang S. Design of a control system for scanning movement of ICT. Manufacturing Automation,1995,17(3) : 44-49)

[5] Yan Lei,Zhao Gang,Ryu Si-Heon,eta1. The platform of image acquisition and processing system based on DSP and FPGA[J]. ICSMA 2008-International Conference on Smart Manufacturing Application,2008 : 470—473.

[6] fang Shujie,Guo Zonglian. Development of hish—speed data acquisition system based on FPGA[J]. Dianli Zidonghua Shebei/Electric Power Automation Equipment,2008,28(6) : 93—96.

[7] Slootweg J GWind Power Modeling and Impact on Power System Dynamics,PHD Thesis[D]. Ridderpdnt Offset drukkerijB. VRidderkerk. Netherlands : 123-130.

[8] WU Xue-guang. eta1. Application of Models of the Wind Energy Conversion System to Wind Power Dynamic Analysis[A]. in : Proceedings POWERCON 98. 